\title{
Radioimmunotherapy Improves Survival of Rats with Microscopic Liver Metastases of Colorectal Origin
}

\author{
Gabie M. de Jong, MD ${ }^{1}$, Thijs Hendriks, $\mathrm{PhD}^{1}$, Annemarie Eek, $\mathrm{Msc}^{2}$, Wim J. G. Oyen, $\mathrm{PhD}^{2}$, Sandra Heskamp, \\ $\mathbf{M s c}^{2}$, Robert P. Bleichrodt, $\mathbf{P h D}^{1}$, and Otto C. Boerman, $\mathbf{P h D}^{2}$ \\ ${ }^{1}$ Department of Surgery, Division of Abdominal and Oncological Surgery, Radboud University Nijmegen Medical Centre, \\ Nijmegen, The Netherlands; ${ }^{2}$ Department of Nuclear Medine, Radboud University Nijmegen Medical Centre, Nijmegen, \\ The Netherlands
}

\begin{abstract}
Background. Half of the patients with colorectal cancer develop liver metastases during the course of their disease. The aim of the present study was to assess the efficacy of radioimmunotherapy (RIT) with a radiolabeled monoclonal antibody $(\mathrm{mAb})$ to treat experimental colorectal liver metastases.
\end{abstract}

Methods. Male Wag/Rij rats underwent a minilaparotomy with intraportal injection of $1 \times 10^{6}$ CC531 tumor cells. The biodistribution of ${ }^{111}$ In-labeled MG1, 1 day after intravenous administration, was determined in vivo and compared with that of an isotype-matched control antibody (UPC-10). The maximal tolerated dose (MTD) of ${ }^{177} \mathrm{Lu}-$ labeled MG1 was determined and the therapeutic efficacy of ${ }^{177} \mathrm{Lu}-\mathrm{MG} 1$ at MTD was compared with that of ${ }^{177} \mathrm{Lu}-$ UPC-10 and saline only. RIT was administered either at the day of tumor inoculation or 14 days after tumor inoculation. Primary endpoint was survival.

Results. ${ }^{111}$ In-MG1 preferentially accumulated in CC531 liver tumors $(9.2 \pm 3.7 \% \mathrm{ID} / \mathrm{g})$, whereas ${ }^{111} \mathrm{In}-\mathrm{UPC}-10 \mathrm{did}$ not $(0.8 \pm 0.1 \% \mathrm{ID} / \mathrm{g})$. The MTD of ${ }^{177} \mathrm{Lu}-\mathrm{MG} 1$ was $400 \mathrm{MBq} / \mathrm{kg}$ body weight. Both the administration of ${ }^{177}$ Lu-MG1 and ${ }^{177}$ Lu-UPC-10 had no side-effects except a transient decrease in body weight. The survival curves of the group that received ${ }^{177} \mathrm{Lu}-\mathrm{UPC}-10$ and the group that received saline only did not differ $(P=0.407)$. Administration of ${ }^{177}$ Lu-MG1 RIT immediately after surgery improved survival significantly compared with

(C) The Author(s) 2009. This article is published with open access at Springerlink.com

First Received: 19 January 2009;

Published Online: 9 May 2009

G. M. de Jong, MD

e-mail: g.dejong@chir.umcn.nl administration of ${ }^{177}$ Lu-UPC-10 $(P=0.009) \quad$ whereas delayed treatment did not $(P=0.940)$.

Conclusion. This study provides proof of principle that RIT can be an effective treatment modality for microscopic liver metastases, whereas RIT is not effective in larger tumors.

Colorectal cancer (CRC) is the third most common cancer affecting both men and women in the Western world. $^{1,2}$ CRC has a worldwide 5-year survival of 40-60\% and prognosis is mainly determined by the presence of local or distant metastases. ${ }^{3}$ The liver mostly is the first site of haematogenous dissemination of CRC. Twenty-five percent of the patients have liver metastases at the time of presentation and another $25 \%$ of the patients with CRC develop liver metastases within 2 years after surgical resection. ${ }^{4,5}$ Development of these metachronous liver metastases is hypothesized to be the result of circulating tumor cells or activation of dormant microscopic tumors in the liver. A second hypothesis is that small liver tumors remain undetected at the time of diagnosis due to the limited sensitivity of imaging techniques. ${ }^{6}$ For these reasons, patients with stage III colon cancer or high-risk patients with stage II colon cancer will receive adjuvant treatment with 5-fluorouracil (5-FU)-based chemotherapy. ${ }^{7}$ However, pyrimidine-antagonists, such as 5-FU, induce inherent adverse events and the beneficial effect of adjuvant chemotherapy for stage II patients is still subject of debate. $^{8}$

Radioimmunotherapy (RIT) using radiolabeled monoclonal antibodies directed against tumor-associated antigens offers the opportunity to selectively irradiate tumor cells, while sparing normal tissues. Several preclinical studies have indicated that RIT can be very 
effective and is at least equivalent to chemotherapy. ${ }^{9-12}$ Tumor targeting with radiolabeled monoclonal antibodies has been shown to be particularly efficient in small-volume disease and minimal residual disease. ${ }^{11,13}$ The aim of the present study was to test the hypothesis that RIT might be an effective treatment modality to treat microscopic and small liver tumors.

\section{MATERIALS AND METHODS}

The in vivo experiments were performed in rats using a MG1 monoclonal antibody (mAb) directed against a syngeneic colon carcinoma cell line (CC531). Sequential experiments were performed to optimize and demonstrate the feasibility of RIT in this animal model. First, the internalization kinetics of the MG1 binding to its antigen were determined. Secondly, the maximum tolerated dose (MTD) of ${ }^{177}$ Lu-MG1 was determined. Next, the biodistribution of ${ }^{111}$ In-labeled MG1 was examined in Wag/Rij rats with CC531 liver tumors and compared with the biodistribution of an isotype-matched irrelevant control antibody (UPC-10). Subsequently, the therapeutic efficacy of RIT using ${ }^{177}$ Lu-labeled MG1 to treat liver metastases was tested in two disease stages.

\section{Cell Line and Monoclonal Antibody}

The syngeneic rat colonic carcinoma cell line CC531 is derived from colonic tumors of Wag/Rij rats exposed to 1,2-dimethylhydrazine. ${ }^{14}$ CC531 was cultured and suspended as described previously. ${ }^{15}$ MG1 mAb was purchased from Antibodies for Research Applications BV (Gouda, The Netherlands). The MG1 mAb is a murine $\mathrm{IgG}_{2 \mathrm{a}}$ antibody, raised by immunization of mice with CC531 colon carcinoma cells. It recognizes a cell surface antigen of about $80 \mathrm{kDa}$, localizes preferentially in the CC531 tumors when injected in rats, and has minimal cross-reactivity with other cell types. ${ }^{16,17}$ MG1 has a high affinity $\left(K_{\mathrm{d}}=1.0 \mathrm{nM}\right)$ for the antigen expressed on CC531 tumor cells. ${ }^{18}$ UPC-10 is also a murine $\operatorname{IgG}_{2 \mathrm{a}}$ antibody and is directed against $\beta$-2-6-linked fructosan (Sigma Life Science, Zwijndrecht, The Netherlands).

\section{Radiolabeling}

\footnotetext{
${ }^{177} \mathrm{Lu}$ is a beta-emitting radionuclide with a maximum beta-energy of $0.497 \mathrm{MeV}$ and a half-life of 6.7 days that is commonly used in radioimmunotherapy. ${ }^{111}$ In has highly similar physical characteristics to ${ }^{177} \mathrm{Lu}$ but does not emit beta-particles and was therefore used as surrogate radionuclide for ${ }^{177} \mathrm{Lu}$ in in vitro and biodistribution experiments. ${ }^{111}$ In was purchased from Covidien BV
}

(Petten, The Netherlands) and ${ }^{177}$ Lu from IDB Holland BV (Baarle Nassau, The Netherlands). ${ }^{111}$ In- and ${ }^{177}$ Lu-labeling of the MG1-DTPA antibody conjugate was performed and radiochemical purity and immunoreactivity were confirmed as described previously. ${ }^{15}$ Specific activity of the ${ }^{111}$ In-MG1 and ${ }^{111}$ In-UPC-10 preparations was $218 \mathrm{kBq} / \mu \mathrm{g}$ and $200 \mathrm{kBq} / \mu \mathrm{g}$, respectively. Specific activity of the ${ }^{177} \mathrm{Lu}-\mathrm{UPC}-10$ preparation was $588 \mathrm{kBq} / \mu \mathrm{g}$. Average specific activity of the ${ }^{177} \mathrm{Lu}-\mathrm{MG} 1$ preparations was $494 \mathrm{kBq} / \mu \mathrm{g}$ (range $396-629 \mathrm{kBq} / \mu \mathrm{g}$ ). The radiochemical purity of all preparations exceeded $90 \%$.

\section{Internalization of $M G 1$}

CC531 cells were cultured to confluency in six-well plates and were incubated with $1.1 \mathrm{kBq}{ }^{111} \mathrm{In}-\mathrm{MG} 1$ for 0.5 , $1,2,4,6$, and $24 \mathrm{~h}$, in $2 \mathrm{ml}$ binding buffer (RPMI 1640, $0.5 \% \mathrm{BSA}$ ), at $37^{\circ} \mathrm{C}$ in a humidified atmosphere with $5 \%$ $\mathrm{CO}_{2}$. After incubation, $1 \mathrm{ml}$ acid wash buffer (0.1 M HAc, $0.15 \mathrm{M} \mathrm{NaCl}, \mathrm{pH} 2.6$ ) was added for $10 \mathrm{~min}$ to remove the membrane-bound fraction of ${ }^{111}$ In-MG1. Subsequently, the cells were harvested from the six-well plates and the amount of activity was measured in a shielded 3-inch-welltype gamma counter (Wizard, Pharmacia-LKB, Sweden). Nonspecific binding and internalization was determined by coincubation with an excess of $6.7 \mathrm{nM}$ unlabeled MG1.

\section{Animals}

Ten- to 12-week-old male Wag/Rij-rats with mean weight of $250 \mathrm{~g}$ were obtained from Charles River Laboratories (Sulzfeld, Germany). Rats were accustomed to laboratory conditions for 1 week prior to experimental use and housed under nonsterile, standard laboratory conditions (temperature $20-24^{\circ} \mathrm{C}$; relative humidity $50-60 \%$; $12 \mathrm{~h}$ light/12 h dark) on sawdust in filter-topped cages (two or three rats per cage) with free access to animal chow (Ssniff Voer, Soest, The Netherlands) and water. All experiments were conducted in accordance with the principles laid out by the revised Dutch Act on Animal Experimentation (1997) and approved by the institutional Animal Welfare Committee of the Radboud University Nijmegen.

\section{Maximum Tolerated Dose}

Fifteen healthy male Wag/Rij rats were injected intravenously with increasing doses of ${ }^{177}$ Lu-MG1 (400, 600 , and $800 \mathrm{MBq} / \mathrm{kg}$, respectively). Five rats were used as controls, receiving an intravenous injection with the saline only. Blood samples were collected twice during the first week and weekly for the following 7 weeks post injection. White blood cell (WBC) counts and platelet 
counts were analyzed by ADVIA 120 Hematology System (Siemens Medical Solutions Diagnostics, Deerfield, USA). Toxicity was evaluated by monitoring the body weight of the animals, general condition, and blood cell counts. When the humane endpoint ( $>20 \%$ body weight loss, physical inactivity or signs of severe toxicity such as infections, bleeding, or diarrhea) was reached, rats were killed using $\mathrm{O}_{2} / \mathrm{CO}_{2}$ asphyxiation and immediately dissected. After 2 months all remaining rats were killed. MTD was defined as the highest activity $(\mathrm{MBq} / \mathrm{kg})$ at which $100 \%$ of the animals survived without reaching the humane endpoint.

\section{Tumor Inoculation}

The surgical procedures to induce intrahepatic CC531 tumors for the biodistribution and therapy studies were carried out under clean conditions. Prior to operation, rats received carprofen subcutaneously ( $5 \mathrm{mg} / \mathrm{kg}$ body weight) as analgesic. Immediately after the onset of general anaesthesia (isoflurane $/ \mathrm{O}_{2} / \mathrm{N}_{2} \mathrm{O}$ ), the abdomen was shaved and disinfected with $70 \%$ ethanol. During the operation, rats were placed on a warm mattress to limit heat loss and chloramphenicol $(20 \mathrm{mg} / \mathrm{g})$ eye drops were administered to prevent dehydration of the eyes. Rats subsequently underwent a $3-\mathrm{cm}$ midline minilaparotomy. After the abdomen was opened, the intestines were gently lifted out of the abdomen, wrapped in moist gauzes, and placed on the left side of the animal, exposing the portal vein. Using a 29gage needle and 1-ml syringe, $1 \times 10^{6}$ CC531 tumor cells, suspended in $0.1 \mathrm{ml}$ phosphate-buffered saline (PBS), were injected intraportally. To prevent excessive bleeding, the portal vein was punctured through mesenteric fat, which served as a tamponade. In addition, the puncture area was covered for a few minutes with a cotton stick. The muscular layer of the abdomen was closed by continuous absorbable braided 3/0 sutures and the skin was closed by iron wound clips. Ten milliliters of warmed saline was injected subcutaneously for rehydration. Rat cages were kept on a warmed mattress for the first day. The rats received daily analgesic subcutaneous injections for 2 days.

\section{Biodistribution}

To determine the uptake of ${ }^{177} \mathrm{Lu}-\mathrm{MG} 1$ in normal tissues and liver tumors, the biodistribution of ${ }^{111} \mathrm{In}-\mathrm{MG} 1$ was determined after intravenous injection $(n=4)$. Likewise, the biodistribution of ${ }^{111}$ In-UPC-10 was determined in a separate group $(n=4)$. In this experiment, on day 0 eight $\mathrm{Wag} / \mathrm{Rij}$ rats underwent the surgical procedure to induce intrahepatic tumor growth as described above. Twenty days later, rats received a single intravenous injection with either $0.4 \mathrm{MBq}{ }^{111} \mathrm{In}-\mathrm{MG} 1$ (1.7 $\mu \mathrm{g}$ MG1 $\mathrm{mAb}$ per rat) or $0.4 \mathrm{MBq}{ }^{111}$ In-UPC-10 (1.9 $\mu \mathrm{g}$ UPC-10 mAb per rat), in $0.2 \mathrm{~mL}$ PBS, $0.5 \%$ bovine serum albumin (BSA). On day 21 the rats were killed using $\mathrm{O}_{2} / \mathrm{CO}_{2}$ asphyxiation and dissected. Uptake in normal tissues and liver tumors was measured as described previously. ${ }^{18}$ The results were expressed as percentage of the injected dose per gram tissue $(\% \mathrm{ID} / \mathrm{g})$.

\section{Radioimmunotherapy}

The therapeutic efficacy of RIT with ${ }^{177}$ Lu-MG1 was determined by the random assignment of 56 rats to receive an intravenous injection of either ${ }^{177} \mathrm{Lu}-\mathrm{MG} 1,{ }^{177} \mathrm{Lu}$-UPC10 or saline $2-4 \mathrm{~h}$ after intraportal injection of CC531 cells $\left(n=14\right.$ each). In a separate group, ${ }^{177} \mathrm{Lu}-\mathrm{MG} 1$ was also administered 14 days after tumor cell inoculation $(n=14)$ as shown in Fig. 1. Figure 2 shows an example of the tumor nodules as present in the liver 14 days after inoculation. In all groups, the administered activity was $300 \mathrm{MBq} / \mathrm{kg}$ in $0.25 \mathrm{ml}$ PBS in a single dose. The primary endpoint was survival. Toxicity was evaluated by monitoring body weight and general condition. Rats were monitored daily by two experienced and independent animal technicians who were blinded to the therapeutic regime. When the humane endpoint was reached, rats were euthanized by $\mathrm{O}_{2} / \mathrm{CO}_{2}$ asphyxiation and immediately dissected. All remaining rats were euthanized and dissected 120 days after the operation. At dissection, the abdominal cavity was carefully inspected, with special attention to the liver parenchyma. All macroscopic tumor deposits in the liver were resected and weighed. When no macroscopic tumor deposits were present, the liver was sliced in $0.5-\mathrm{cm}$ -

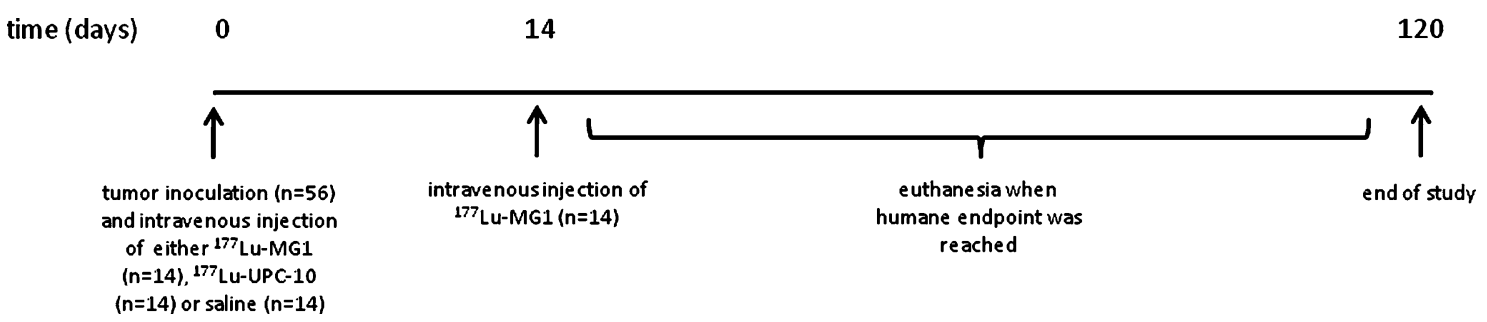

FIG. 1 Time schedule of the radioimmunotherapy experiment 


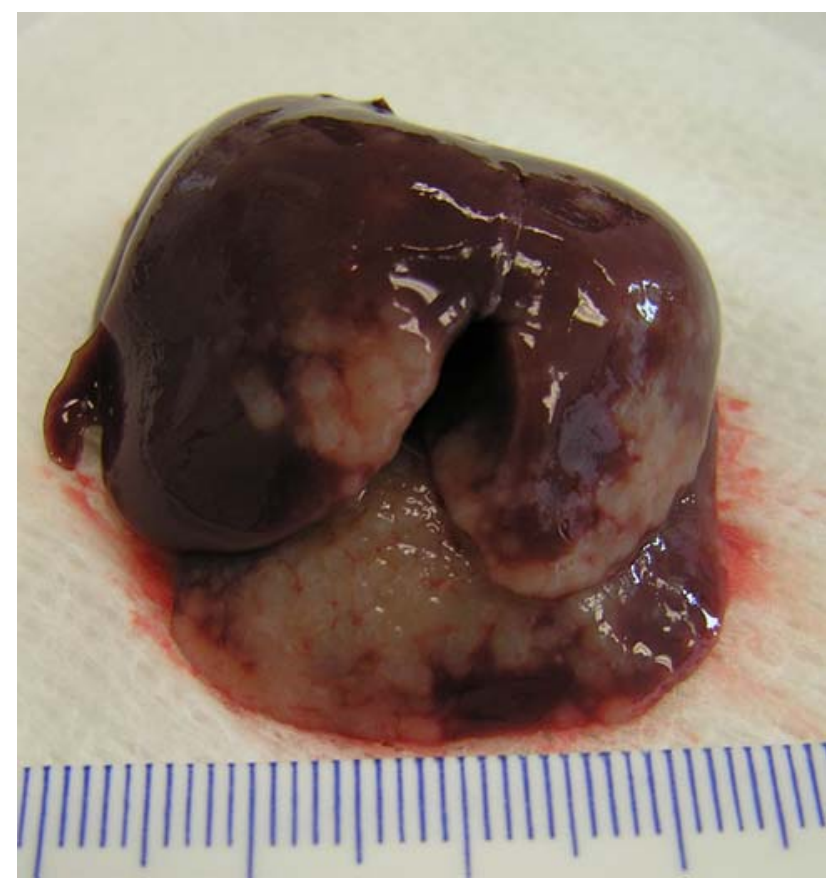

FIG. 2 Anterior site of liver with metastases 14 days after intraportal injection of $1 \times 10^{6}$ CC531 tumor cells

thick slices for routine histopathological hematoxylin and eosin (H\&E) staining. Microscopic tumor growth was scored as either present or absent.

\section{Statistical Analysis}

Statistical analysis was performed using the SPSS software (Chicago, IL) and GraphPad Prism version 4.00 (GraphPad Software, San Diego USA) for Windows. Sample size was determined by a power calculation based on an estimated effect of RIT in doubling the survival time of the treated animals. Multiple comparisons of dichotomous values were analyzed using the one-way analysis of variance (ANOVA)test. Bonferroni correction for multiple testing was applied. Survival time was analyzed by Kaplan-Meier method and differences were tested by GehanBreslow test since no consistent proportional hazard ratio was to be expected. The level of statistical significance was set at $P<0.05$. Medians, 75 th-, and 25th survival percentiles and standard error were used to describe continuous data.

\section{RESULTS}

\section{Internalization of $M G 1$}

After $6 \mathrm{~h}, 13 \pm 1 \%$ of the incubated ${ }^{111}$ In-MG1 was bound to the target cells (Fig. 3). MG1 was internalized

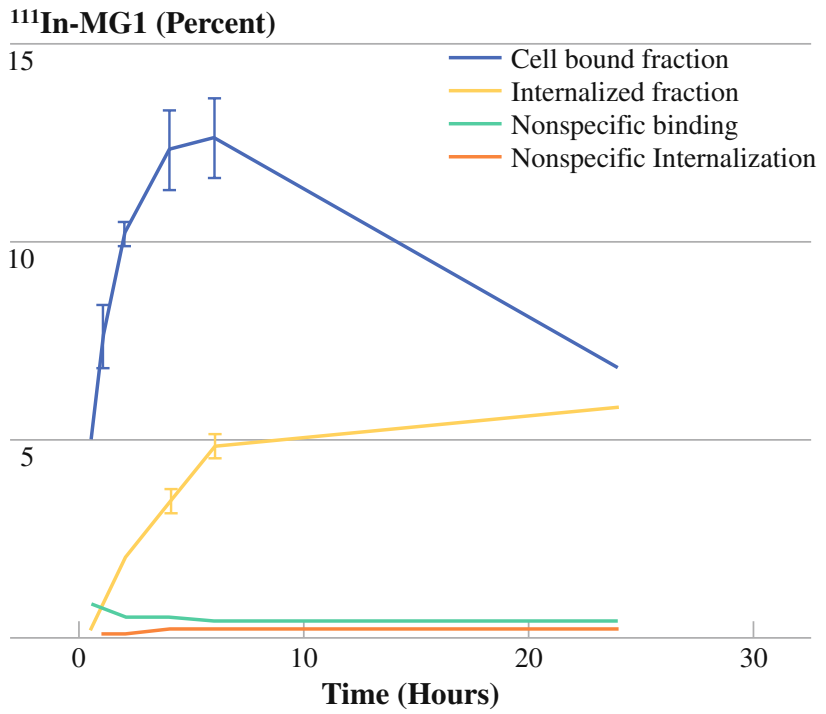

FIG. 3 Internalization of MG1 in the CC531 tumor cell. The percentage activity of ${ }^{111}$ In-MG1 present in the cell bound fraction (blue line) and in the internalized fraction (yellow line) is depicted as mean \pm standard error on the mean (SEM). In addition, nonspecific binding (green line) and internalization (orange line) are given

gradually by the target cells: after $6 \mathrm{~h}, 38 \%$ of the cellassociated ${ }^{111}$ In-MG1 was internalized.

\section{Maximum Tolerated Dose}

Survival of the animals in the MTD study is summarized in Fig. 4. During the first days post injection, all groups receiving ${ }^{177} \mathrm{Lu}-\mathrm{MG} 1$ lost weight. After 18 days, $80 \%$ of the animals that received either $800 \mathrm{MBq} / \mathrm{kg}$ or $600 \mathrm{MBq} /$ $\mathrm{kg}{ }^{177} \mathrm{Lu}-\mathrm{MG} 1$ had lost more than $20 \%$ of their body weight and were euthanized. These rats all showed signs of dehydration and poor general condition. There was no diarrhea. At dissection the thymus could not be detected, the spleen was small, and the intestines appeared fragile. Moreover, seven of these rats had signs of infarction and bleeding in the kidneys, testicles, and around the spleen. The rats that received $400 \mathrm{MBq} / \mathrm{kg}$ lost a maximum of $7 \%$ of their body weight, reaching nadir on day 5 , and showed signs of clinical discomfort such as inactive behavior during this period. The control animals on average gained $3 \%$ of their relative body weight during the corresponding period. After 5 days the animals that had received $400 \mathrm{MBq} / \mathrm{kg}$ started to gain weight again, as the control animals.

WBC and platelet counts are shown in Fig. 5a, b. All groups showed a similar decrease in WBC during the first 14 days post injection, with minimum levels of less than $1 \%$ of the initial value. In all remaining rats, WBC started to recover from day 20 , reaching a normal WBC count 


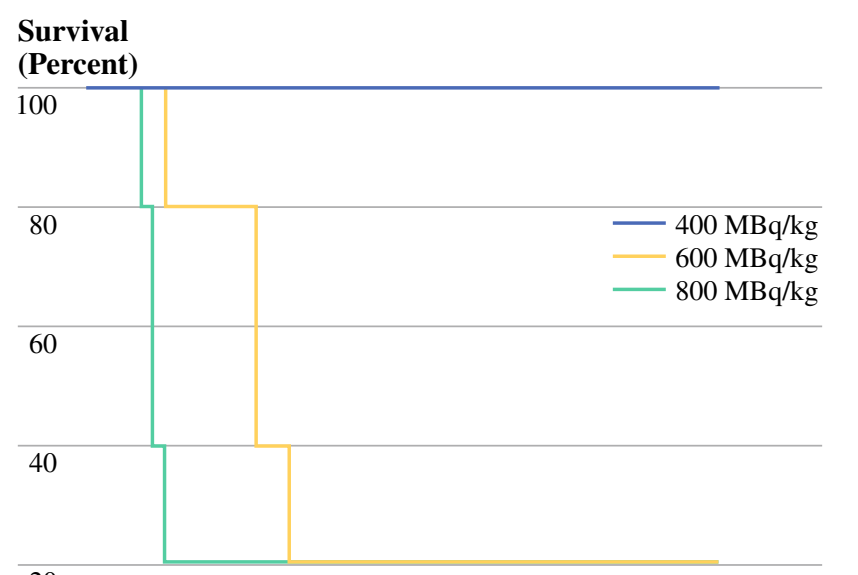

20

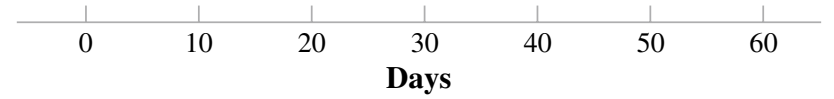

FIG. 4 Survival curves of the animals in the MTD study. Lines represent groups receiving escalating doses of ${ }^{177} \mathrm{Lu}-\mathrm{MG} 1 ; 400 \mathrm{MBq} /$ $\mathrm{kg}$ (blue line), $600 \mathrm{MBq} / \mathrm{kg}$ (yellow line), and $800 \mathrm{MBq} / \mathrm{kg}$ (green line). Note that the control group is not represented in the analyses, since all rats survived the experimental period

after 2 months. Platelets started to decline 4 days after injection and started to recover after 2 weeks.

\section{Biodistribution of ${ }^{111}$ In-Labeled MG1}

The biodistribution of ${ }^{111} \mathrm{In}-\mathrm{MG} 1$ and ${ }^{111} \mathrm{In}-\mathrm{UPC}-10$ in rats with intrahepatic CC531 tumors is summarized in

a

\section{WBC}

$\left(\times 10^{9} / \mathrm{L}\right)$

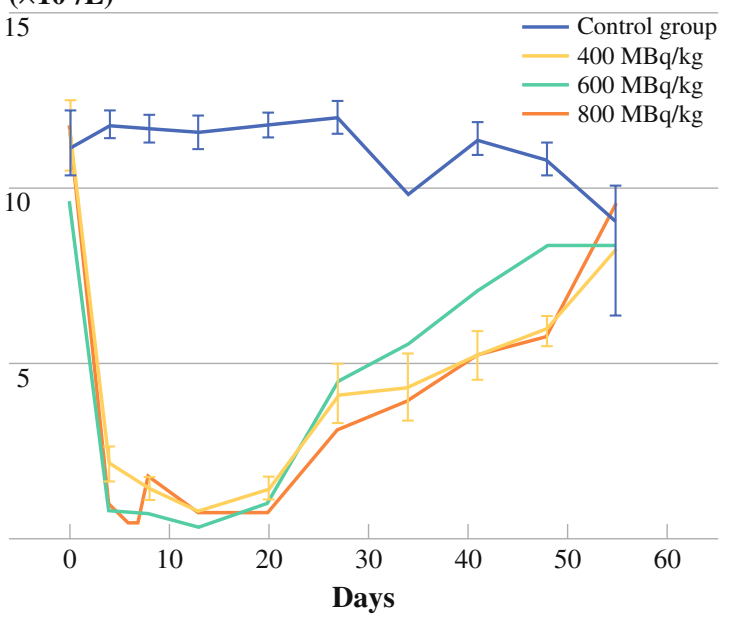

FIG. 5 Hematological toxicity of ${ }^{177}$ Lu-MG1. Both WBC (a) and platelet counts (b) are given. Lines represent groups receiving escalating activities of ${ }^{177} \mathrm{Lu}-\mathrm{MG} 1 ; 400 \mathrm{MBq} / \mathrm{kg}$ (yellow line),
Fig. 6. There was high and specific uptake of ${ }^{111} \mathrm{In}-\mathrm{MG} 1$ in the tumor of $9.2 \pm 3.7 \% \mathrm{ID} / \mathrm{g}$, as compared with the tumor uptake of ${ }^{111}$ In-UPC-10 $(0.8 \pm 0.1 \% \mathrm{ID} / \mathrm{g}) \quad 1$ day post injection. Uptake of MG1 in other organs did not exceed $1.2 \% \mathrm{ID} / \mathrm{g}$. The tumor-to-blood ratio of ${ }^{111} \mathrm{In}-\mathrm{MG} 1$ was $7.1 \pm 1.3$ compared with $0.3 \pm 0.01$ for ${ }^{111} \mathrm{In}-\mathrm{UPC}-10$.

\section{Efficacy of Radioimmunotherapy Using ${ }^{177}$ Lu-MGI}

The relative body weight of the rats during the first 30 days of the study, expressed as the percentage of the body weight on the day of surgery, is depicted in Fig. 7. Compared with administration of the saline only, administration of both ${ }^{177} \mathrm{Lu}-\mathrm{MG} 1$ and ${ }^{177} \mathrm{Lu}-\mathrm{UPC}-10$ reduced body weight, indicating a nonspecific radiation effect. On either time point, 5 days after injection of the radiopharmaceutical, the relative body weight decreased about $4 \%$. However, no other signs of clinical discomfort were observed during the initial posttreatment period, and bodyweight recovered quickly in the course of the study. In addition, no signs of wound healing disorders were observed.

One rat of the group treated with ${ }^{177}$ Lu-MG1 on day 14 was euthanized without completely fulfilling the humane endpoint criteria and was therefore excluded from the survival analysis. Altogether 50 rats reached the humane endpoint in the course of the experiment and were killed. All of these rats, including all rats in the control group, had developed liver tumors at that moment. The mean tumor weight of the group treated with ${ }^{177} \mathrm{Lu}-\mathrm{MG} 1$ on day 14 was

\section{b}

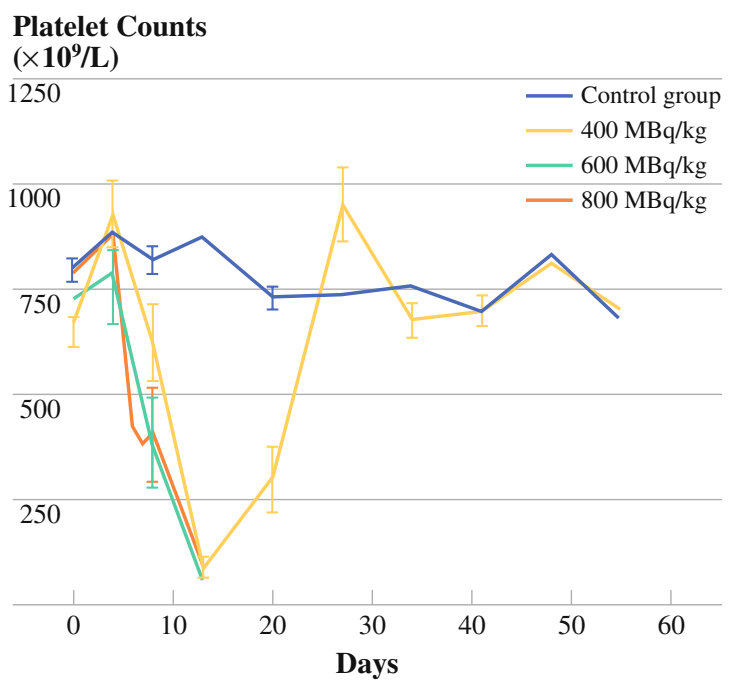

$600 \mathrm{MBq} / \mathrm{kg}$ (green line), and $800 \mathrm{MBq} / \mathrm{kg}$ (orange line) and the control group (blue line). Symbols represent mean values with standard error. No dose-response pattern was seen in these groups 
FIG. 6 Biodistribution. The uptake of ${ }^{111}$ In-DTPA-MG1 (blue line) and ${ }^{111} \mathrm{In}$ DTPA-UPC-10 (yellow line) on day 20 is depicted as mean \pm SEM

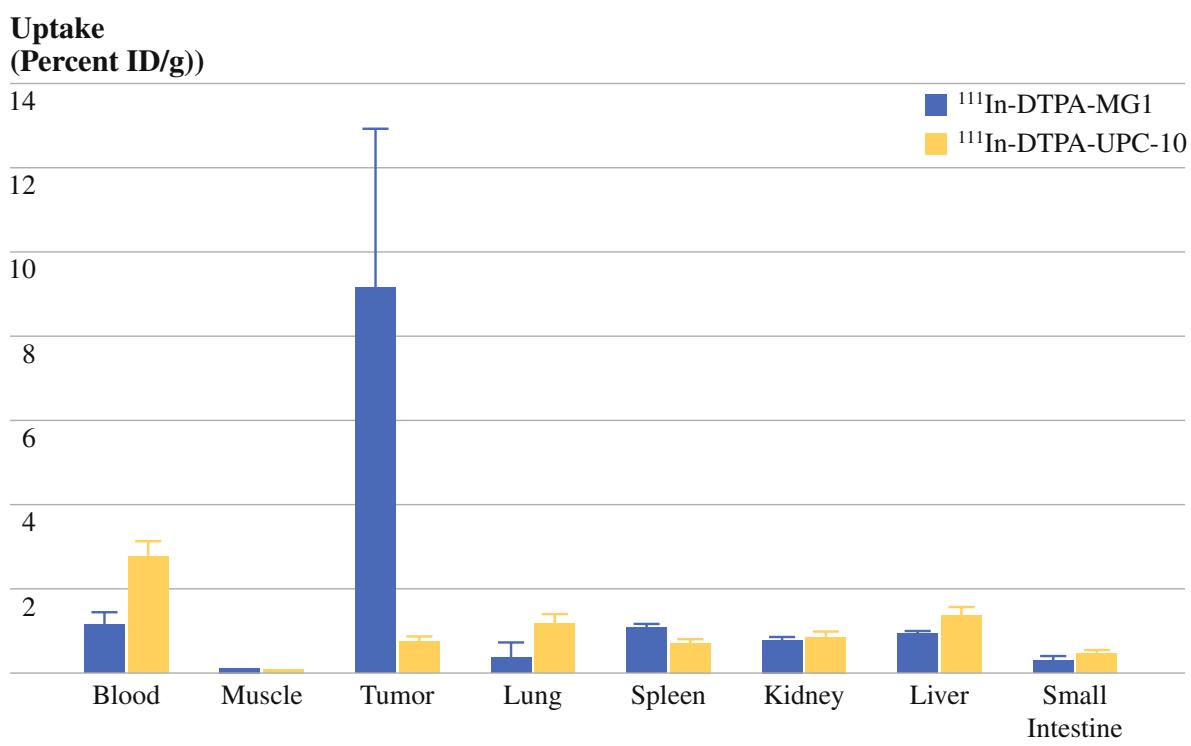

Uptake

(Percent ID/g))

Intestine

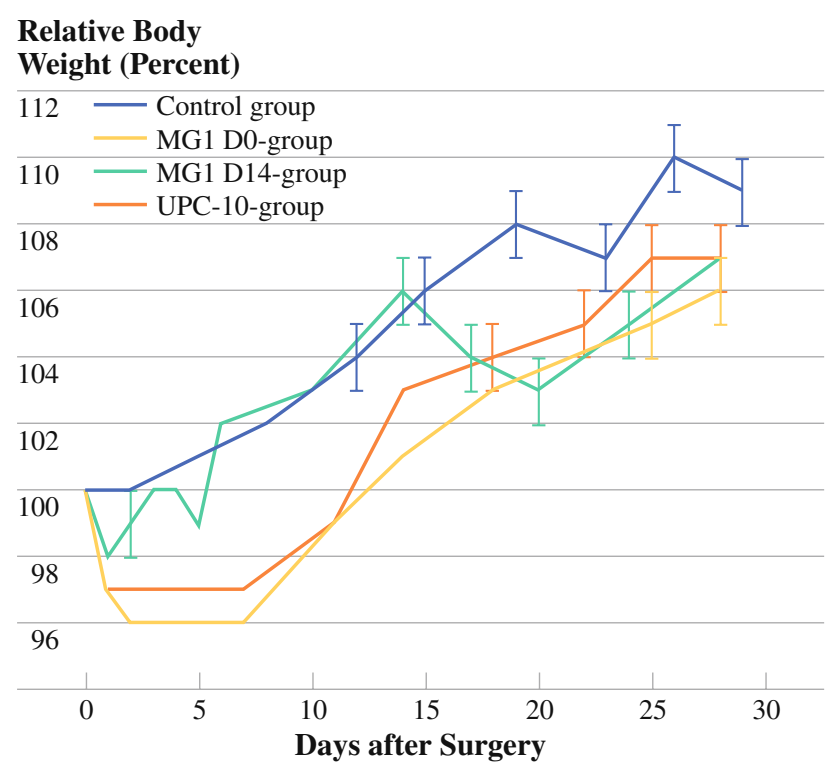

FIG. 7 Course of body weight. The mean $( \pm$ SEM) relative body weight, with respect to preoperative weight, is given for the MG1 D0 group (yellow line), MG1 D14 group (green line), UPC-10 group (orange line), and the control group (blue line)

$15 \pm 1 \mathrm{~g}$ and was significantly higher than the mean tumor weight of each of the other groups $(P<0.05$ for each comparison). Twenty-seven rats also had tumor nodules elsewhere in the abdomen, including at the initial puncture site. One rat of the group treated with ${ }^{177} \mathrm{Lu}-\mathrm{MG} 1$ on day 0 developed jaundice 90 days after start of the experiment and was found to have an obstructing tumor at the liver hilus. One hundred and twenty days after tumor cell inoculation five rats (three in the group that was treated with ${ }^{177}$ Lu-MG1 on day 0 and one each in the groups treated with ${ }^{177} \mathrm{Lu}-\mathrm{UPC}-10{ }^{177}$ on day 0 or ${ }^{177} \mathrm{Lu}-\mathrm{MG} 1$ on

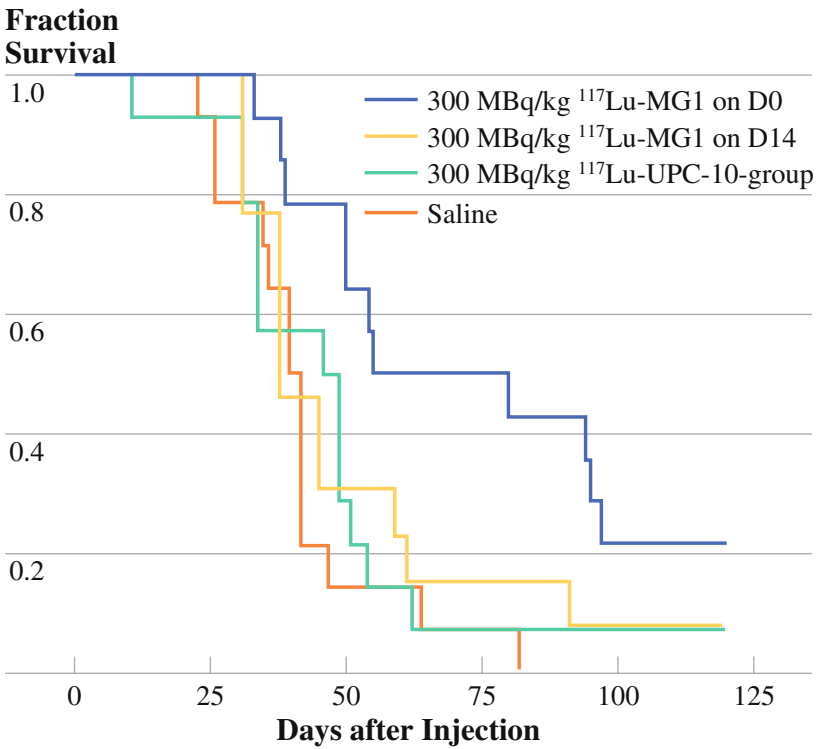

FIG. 8 Survival curves of the animals in the therapy study. Lines represent groups receiving $300 \mathrm{MBq} / \mathrm{kg}{ }^{177} \mathrm{Lu}-\mathrm{MG} 1$ on D0 (yellow line), $300 \mathrm{MBq} / \mathrm{kg}{ }^{177} \mathrm{Lu}-\mathrm{MG} 1$ on $\mathrm{D} 14$ (green line), $300 \mathrm{MBq} / \mathrm{kg}$ ${ }^{177} \mathrm{Lu}$-UPC-10 group (orange lne) or saline (blue line)

day 14) were still alive, without clinical signs of liver tumors. At dissection none of the rats had macroscopic tumor growth. Histopathological examination of the livers showed no microscopic tumor nodules either.

The survival curves are shown in Fig. 8. Statistical analysis showed that the survival curves of the groups receiving ${ }^{177} \mathrm{Lu}-\mathrm{UPC}-10$ or the saline only did not differ $(P=0.518)$. Therefore, in further analysis, the results were compared with the group receiving ${ }^{177} \mathrm{Lu}$-UPC-10. First, Kaplan-Meier survival analysis was performed, which showed that overall survival curves of the groups treated 
with ${ }^{177} \mathrm{Lu}-\mathrm{MG} 1$ and ${ }^{177}$ Lu-UPC-10 were significantly different $(P=0.029)$. Administration of RIT immediately after surgery improved survival significantly compared with administration of the control antibody $(P=0.009)$ and compared with delayed treatment $(P=0.027)$. Administration of RIT 14 days after tumor inoculation did not improve survival compared with administration of the control antibody $(P=0.940)$. The median survival of the animals treated with ${ }^{177} \mathrm{Lu}$-UPC-10 was $46 \pm 6$ days. The 75th and 25th survival percentiles of this group were $34 \pm 9$ days and $49 \pm 3$ days, respectively. The median survival of the rats treated with ${ }^{177} \mathrm{Lu}-\mathrm{MG} 1$ on the day of surgery was $55 \pm 24$ days and the $75^{\text {th }}$ and $25^{\text {th }}$ survival percentile of this group improved to $50 \pm 7$ days and $97 \pm 1$ days, respectively.

\section{DISCUSSION}

The results of the present study show that RIT can increase survival in rats with microscopic tumors of colorectal origin in the liver. A well-characterized rat model was used in the study. The syngeneic rat colon carcinoma cell line CC531 exhibits a reproducible growth pattern in Wag/Rij rats. ${ }^{19}$ We already demonstrated that MG1 has a high affinity for its antigen on the CC531 cell membrane. ${ }^{18}$ In addition, the present data demonstrate that MG1 is gradually internalized by CC531 cells, which is a favorable characteristic for RIT since it increases tumor retention of the radiolabel and consequently enhances the radiation dose absorbed by the tumor. The remarkable decrease in cell surface binding of the labeled antibody between 6 and $24 \mathrm{~h}$ might indicate either release of the radiolabeled $\mathrm{mAb}$ from the cell surface or shedding of the antibody-antigen complex after internalization. Both intrasplenic and intraportal injection of (syngeneic) tumor cells in rodents may result in liver metastases with a high reproducibility. ${ }^{20} \mathrm{In}$ the present model, tumor growth in the liver occurs in $100 \%$ of the untreated animals, with no mortality or weight loss due to the surgical procedure. The in vivo tumor targeting potential of MG1 mAb for CC531 liver tumor is demonstrated by its specific accumulation in CC531 liver tumors and is in concordance with findings in previous animal studies on peritoneal carcinomatosis. ${ }^{15,16,19}$ In addition, the MTD of ${ }^{177} \mathrm{Lu}-\mathrm{MG} 1$ for healthy male Wag/rij rats was found to be approximately $400 \mathrm{MBq} / \mathrm{kg}$ with doselimiting hematological toxicity.

Currently, adjuvant treatment strategies after surgery focus on the rate of dissemination at the time of diagnosis. For patients with stage III CRC, adjuvant 5-FU-based chemotherapy after resection of the primary tumor is most often recommended to improve disease-free survival. ${ }^{7}$ However, pyrimidine antagonists, such as 5-FU, have inherent adverse events. Adjuvant systematic or regional therapies after surgery are also hypothesized to decrease relapse rates in patients with stage II colon cancer. However, a recent systematic review by Figueredo et al. showed that adjuvant systemic 5-FU-based chemotherapy has only small benefit for patients with stage II CRC and should therefore only be considered in a carefully selected group. Regional adjuvant chemotherapy (by portal vein infusion) has shown similar results, but is mostly abandoned because of the technical difficulties of the procedure. As for specific immunotherapy, the authors report enhanced recurrencefree survival, but data on overall survival are lacking due to the short follow-up period. Moreover, some of these treatments (for example, active specific immunotherapy with autologous tumor cell vaccines) are complicated procedures, requiring a dedicated laboratory to obtain an active vaccine and a responsive host. Finally, reports on other adjuvant treatments have been limited and did not show significant beneficial effects. ${ }^{8}$ RIT with radiolabeled monoclonal antibodies directed against tumor-associated antigens has evolved from an appealing concept to one of the standard treatment options for patients with nonHodgkin's lymphoma. ${ }^{21,22}$ RIT offers the opportunity to selectively irradiate tumor cells, while sparing normal tissues. Another advantage of RIT is the crossfire effect, which means that nontargeted cells are irradiated due to radiolabeled antibody uptake by surrounding cells. Because of the relative radiosensitivity of colorectal carcinoma and the availability of mAbs against CRC-associated antigens, the efficacy of RIT using radiolabeled mAbs against CRC has been investigated in various preclinical clinical studies. For example, Behr et al. demonstrated that, in a nudemouse model for lung metastases of CRC, RIT $\left({ }^{131}\right.$ I-antiCEA antibody) cured half of the animals whereas equitoxic chemotherapy only prolonged life for a few weeks. ${ }^{23}$ Aarts et al. demonstrated that adjuvant RIT $\left({ }^{177} \mathrm{Lu}-\mathrm{MG} 1 \mathrm{mAb}\right)$ after debulking of peritoneal carcinomatosis in a rat model improved survival, whereas adjuvant heated intraperitoneal chemotherapy did not and was considered more toxic. ${ }^{12}$ To date, only a limited number of clinical trials have studied the effect of RIT in CRC patients. A review of this small number of phase I/II RIT trials in patients with gross metastatic disease precludes any firm conclusion on the potential of RIT in the treatment of CRC. ${ }^{24}$ Most phase I/II clinical RIT studies were carried out in pretreated patients with bulky disease, while it has been shown that RIT may be more effective in patients with small-volume disease. So far, only one study investigated the efficacy of adjuvant RIT in patients with minimal disseminated disease. ${ }^{25}$ In this phase II study 21 patients with small-volume metastatic disease $(\leq 3 \mathrm{~cm})$, all either chemorefractory or chemointolerant, were included. Fourteen patients had liver metastases; remaining patients had either lung metastases 
or peritoneal carcinomatosis. Patients received a single injection of ${ }^{131} \mathrm{I}-\mathrm{MN} 14 \mathrm{IgG}$ at $2.2 \mathrm{GBq} / \mathrm{m}^{2}$ (MTD) and follow-up was as long as 36 months. The therapeutic infusion was tolerated well by all patients, with mild nausea as the only acute side-effect and only mild leucoand thrombocytopenia. Response was measured in 11 out of 21 patients $(52 \%)$, which is similar to response rates achieved with 5-FU/leucovorin-based chemotherapeutic regimens.

Our results support the suggestion that RIT could be efficacious in CRC patients with small liver tumors that cannot be diagnosed at the time of surgery of the primary tumor and constitute an enhanced risk of disease recurrence. The survival analysis of this study shows that immediate administration of adjuvant RIT will mainly result in an improvement of late survival. The data suggest that RIT delays tumor growth in early stages of metastatic development, but does not seem to affect the number of cures and is not effective in animals with larger liver tumors, as demonstrated in the group receiving RIT 14 days after tumor inoculation. For as yet unknown reasons the tumor weight in this group was found to be higher than in the other groups. One rat that was treated with the radiolabeled irrelevant antibody (UPC-10) did not develop any liver tumors. Most likely, this rat did not receive the proper tumor cell inoculation at the start of the experiment. The marked difference in survival between early and late administration of RIT could very well be the result of the inverse correlation between uptake and consequent therapeutic efficacy of radiolabeled antibodies and tumor size. ${ }^{26,27}$ The growth of minimal disease into larger tumors has shown to have a negative effect on the therapeutic efficacy of radiolabeled mAbs. ${ }^{28}$ These conclusions are supported by the outcome of clinical studies demonstrating that tumor targeting with radiolabeled monoclonal antibodies is particularly efficient in small-volume disease and minimal residual disease. ${ }^{23,25}$ Moreover, the current study shows that RIT can be administered intravenously in a single dose and does not show major side-effects such as infections or impaired wound healing. The latter is in line with the findings of Aarts et al., who observed that RIT did not impair wound healing. ${ }^{29}$

If application of RIT is considered in a clinical setting, where surgery-related complications could require intensive care for the patient, special care should be taken to ensure minimal exposure of medical personnel to radiation. The most promising radionuclides for adjuvant RIT in CRC are beta-emitting isotopes such as ${ }^{131} \mathrm{I},{ }^{90} \mathrm{Y}$, and ${ }^{177} \mathrm{Lu} .{ }^{30}$ An important advantage of using ${ }^{177} \mathrm{Lu}$ for RIT (as compared with ${ }^{131} \mathrm{I}$ ) in a clinical setting is the low abundance of gamma radiation $(11 \%, 208 \mathrm{keV})$, lowering the radiation burden to the environment and allowing diagnostic imaging after a therapeutic dose RIT has been administered to a patient.

\section{CONCLUSION}

This study provides proof of principle that RIT using ${ }^{177}$ Lu-labeled MG1 mAb at MTD increases survival in rats with microscopic liver metastases and encourages the implementation of clinical trials studying the effect of RIT in CRC patients undergoing surgery and at high risk for disease recurrence. Further preclinical studies will be performed in this well-defined animal model to optimize the use of (adjuvant) RIT in the treatment of CRC and to compare these effects with the treatment of CRC with adjuvant chemotherapy.

ACKNOWLEDGEMENT The authors wish to thank Roger M. L. M. Lomme, Department of Surgery, Bianca A. M. G. Lemmers - van de Weem and Kitty J.H. Lemmens-Hermans, The Central Animal Laboratory, Radboud University Nijmegen Medical Centre, for excellent technical assistance. Prof. Dr. J.H.J.M. van Krieken, Department of Pathology, Radboud University Nijmegen Medical Centre, is acknowledged for analysis of the histopathological specimens.

OPEN ACCESS This article is distributed under the terms of the Creative Commons Attribution Noncommercial License which permits any noncommercial use, distribution, and reproduction in any medium, provided the original author(s) and source are credited.

\section{REFERENCES}

1. National Cancer Institute. http://www.cancer.gov/. Assessed 13 June 2007.

2. Jemal A, Siegel R, Ward E, Murray T, Xu J, Thun MJ. Cancer statistics. CA Cancer J Clin. 2007;57(1):43-66.

3. Parkin DM. Global cancer statistics in the year 2000. Lancet Oncol. 2001;2(9):533-43.

4. Scheele J, Stangl R, tendorf-Hofmann A. Hepatic metastases from colorectal carcinoma: impact of surgical resection on the natural history. Br J Surg. 1990;77(11):1241-6.

5. Millikan KW, Staren ED, Doolas A. Invasive therapy of metastatic colorectal cancer to the liver. Surg Clin North Am. 1997;77(1):27-48.

6. Bipat S, van Leeuwen MS, Comans EF, Pijl ME, Bossuyt PM, Zwinderman $\mathrm{AH}$, et al. Colorectal liver metastases: CT, MR imaging, and PET for diagnosis-meta-analysis. Radiology. 2005;237(1):123-31.

7. Moertel CG, Fleming TR, Macdonald JS, Haller DG, Laurie JA, Goodman PJ, et al. Levamisole and fluorouracil for adjuvant therapy of resected colon carcinoma. N Engl J Med. 1990;322(6): 352-8.

8. Figueredo A, Coombes ME, Mukherjee S. Adjuvant therapy for completely resected stage II colon cancer. Cochrane Database Syst Rev. 2008;(3):CD005390.

9. Behr TM, Salib AL, Liersch T, Behe M, Angerstein C, Blumenthal RD, et al. Radioimmunotherapy of small volume disease of colorectal cancer metastatic to the liver: preclinical evaluation in comparison to standard chemotherapy and initial results of a phase I clinical study. Clin Cancer Res. 1999;5(10 suppl):3232s-42s.

10. Blumenthal RD, Sharkey RM, Natale AM, Kashi R, Wong G, Goldenberg DM. Comparison of equitoxic radioimmunotherapy and chemotherapy in the treatment of human colonic cancer xenografts. Cancer Res. 1994;54(1):142-51. 
11. Koppe MJ, Soede AC, Pels W, Oyen WJ, Goldenberg DM, Bleichrodt RP, et al. Experimental radioimmunotherapy of small peritoneal metastases of colorectal origin. Int J Cancer. 2003; 106(6):965-72.

12. Aarts F, Hendriks T, Boerman OC, Koppe MJ, Oyen WJ, Bleichrodt RP. A comparison between radioimmunotherapy and hyperthermic intraperitoneal chemotherapy for the treatment of peritoneal carcinomatosis of colonic origin in rats. Ann Surg Oncol. 2007;14(11):3274-82.

13. Behr TM, Goldenberg DM, Becker WS. Radioimmunotherapy of solid tumors: a review "of mice and men". Hybridoma. 1997; 16(1):101-7.

14. Zedeck MS. A model system for studies of colon carcinogenesis: tumor induction by a single injection of methylazoxymethanol acetate. J Natl Cancer Inst. 1974;53:1419-21.

15. Koppe MJ, Hendriks T, Boerman OC, Oyen WJ, Bleichrodt RP. Radioimmunotherapy is an effective adjuvant treatment modality after cytoreductive surgery of peritoneal carcinomatosis of colonic origin. J Nucl Med. 2006;47(11):1867-74.

16. Hagenaars M, Koelemij R, Ensink NG, van Eendenburg JD, van Vlierberghe RL, Eggermont AM, et al. The development of novel mouse monoclonal antibodies against the CC531 rat colon adenocarcinoma. Clin Exp Metastasis. 2000;18(4):281-9.

17. Hagenaars M, Ensink NG, Basse PH, Hokland M, Nannmark U, Eggermont AM, et al. The microscopic anatomy of experimental rat CC531 colon tumor metastases: consequences for immunotherapy? Clin Exp Metastasis. 2000;18(2):189-96.

18. de Jong GM, Boerman OC, Heskamp S, Aarts F, Bleichrodt RP, Hendriks T. Radioimmunotherapy prevents local recurrence of colon cancer in rats. Br J Surg. 2008;96:314-21.

19. Lopes Cardozo AM, Gupta A, Koppe MJ, Meijer S, van Leeuwen PA, Beelen RJ, et al. Metastatic pattern of CC531 colon carcinoma cells in the abdominal cavity: an experimental model of peritoneal carcinomatosis in rats. Eur J Surg Oncol. 2001;27(4): 359-63.

20. de Jong GM, Aarts F, Hendriks T, Boerman OC, Bleichrodt RP. Animal models for liver metastases of colorectal cancer: research review of preclinical studies in rodents. J Surg Res. 2008 (Epub ahead of print).

21. Goldenberg DM. The role of radiolabeled antibodies in the treatment of non-Hodgkin's lymphoma: the coming of age of radioimmunotherapy. Crit Rev Oncol Hematol. 2001;39(1-2): 195-201.
22. Wiseman GA, White CA, Sparks RB, Erwin WD, Podoloff DA, Lamonica $\mathrm{D}$, et al. Biodistribution and dosimetry results from a phase III prospectively randomized controlled trial of Zevalin radioimmunotherapy for low-grade, follicular, or transformed B-cell non-Hodgkin's lymphoma. Crit Rev Oncol Hematol. 2001;39(1-2):181-94.

23. Behr TM, Memtsoudis S, Vougioukas V, Liersch T, Gratz S, Schmidt F, et al. Radioimmunotherapy of colorectal cancer in small volume disease and in an adjuvant setting: preclinical evaluation in comparison to equitoxic chemotherapy and initial results of an ongoing phase-I/II clinical trial. Anticancer Res. 1999;19(4A):2427-32.

24. Koppe MJ, Bleichrodt RP, Oyen WJ, Boerman OC. Radioimmunotherapy and colorectal cancer. Br J Surg. 2005;92(3):264-76.

25. Behr TM, Liersch T, Greiner-Bechert L, Griesinger F, Behe M, Markus PM, et al. Radioimmunotherapy of small-volume disease of metastatic colorectal cancer. Cancer. 2002;94(4 suppl):1373-81.

26. Jain RK. Physiological barriers to delivery of monoclonal antibodies and other macromolecules in tumors. Cancer Res. 1990;50(3 suppl):814s-9s.

27. Brouwers AH, Buijs WC, Mulders PF, de Mulder PH, van den Broek WJ, Mala C, et al. Radioimmunotherapy with [131I]cG250 in patients with metastasized renal cell cancer: dosimetric analysis and immunologic response. Clin Cancer Res. 2005;11(19 Pt 2):7178s-86s.

28. Aarts F, Koppe MJ, Hendriks T, van Eerd JE, Oyen WJ, Boerman $\mathrm{OC}$, et al. Timing of adjuvant radioimmunotherapy after cytoreductive surgery in experimental peritoneal carcinomatosis of colorectal origin. Ann Surg Oncol. 2007;14(2):533-40.

29. Aarts F, Bleichrodt RP, de Man B, Lomme R, Boerman OC, Hendriks T. The effects of adjuvant experimental radioimmunotherapy and hyperthermic intraperitoneal chemotherapy on intestinal and abdominal healing after cytoreductive surgery for peritoneal carcinomatosis in the rat. Ann Surg Oncol. 2008;15(11): 3299-307.

30. Koppe MJ, Bleichrodt RP, Soede AC, Verhofstad AA, Goldenberg $\mathrm{DM}$, Oyen WJ, et al. Biodistribution and therapeutic efficacy of $\left({ }^{125} / 131\right) \mathrm{I}-, \quad\left({ }^{186}\right)$ Re-, $\quad\left({ }^{88} / 90\right) \mathrm{Y}-$, or $\left({ }^{177}\right)$ Lu-labeled monoclonal antibody MN-14 to carcinoembryonic antigen in mice with small peritoneal metastases of colorectal origin. J Nucl Med. 2004;45(7): 1224-32. 